University of Nebraska - Lincoln

DigitalCommons@University of Nebraska - Lincoln

Timothy J. Gay Publications

Research Papers in Physics and Astronomy

$7-1-2001$

\title{
A theoretical study of the effects of insulators on electron transport through electrostatic tube lenses
}

T. G. Anderson

University of Nebraska - Lincoln

B. G. Birdsey

University of Nebraska - Lincoln

S. M. Woeher

University of Nebraska - Lincoln

Mark A. Rosenberry

University of Nebraska-Lincoln, mrosenberry@siena.edu

Timothy J. Gay

University of Nebraska - Lincoln, tgay1@unl.edu

Follow this and additional works at: https://digitalcommons.unl.edu/physicsgay

Part of the Physics Commons

Anderson, T. G.; Birdsey, B. G.; Woeher, S. M.; Rosenberry, Mark A.; and Gay, Timothy J., "A theoretical study of the effects of insulators on electron transport through electrostatic tube lenses" (2001). Timothy J. Gay Publications. 44.

https://digitalcommons.unl.edu/physicsgay/44

This Article is brought to you for free and open access by the Research Papers in Physics and Astronomy at DigitalCommons@University of Nebraska - Lincoln. It has been accepted for inclusion in Timothy J. Gay Publications by an authorized administrator of DigitalCommons@University of Nebraska - Lincoln. 


\title{
A theoretical study of the effects of insulators on electron transport through electrostatic tube lenses
}

\author{
T. G. Anderson, ${ }^{\text {a) }}$ B. G. Birdsey, S. M. Woeher, M. A. Rosenberry, and T. J. Gay \\ Behlen Laboratory of Physics, University of Nebraska, Lincoln, Nebraska 68588-0111
}

(Received 21 August 2000; accepted for publication 17 January 2001)

\begin{abstract}
Using the computer program SIMION 6, we have studied the effects of spurious insulator charging on the trajectories of electrons through electrostatic tube lenses. We considered lens elements with flat ends, spaced a distance $g$ apart, whose inner diameter $D=10 \mathrm{~g}$. For the standard cases of drift tubes, two-element lenses, and einzel lenses, we found that charging effects are eliminated if $t / g>3.5$, where $t$ is the distance between the lens inner diameter and the charged spacing insulator. (C) 2001 American Institute of Physics. [DOI: 10.1063/1.1376654]
\end{abstract}

\section{INTRODUCTION}

Electrostatic tube lenses are often used to transport electrons from their source to a target. The construction of such lens systems typically involves ceramic or plastic insulators that serve to isolate the lens elements electrically and/or align them mechanically. ${ }^{1}$ These insulators are often "charged up" by stray electrons in the apparatus, and develop unpredictable surface potentials. If not shielded from the primary electron beam, the resultant stray electric fields can cause random, sporadic fluctuations of the electron trajectories through the lenses, making beam handling very difficult.

A standard mechanical configuration for tube lenses involves a U-shaped tray containing two insulating rods that support and align the electrically isolated tubes. Alternate designs involve concentric cylindrical insulating spacers or insulating balls seated in alignment holes. In these situations, spurious insulator fields in the beam transport volume are minimized either by staggering the termination of individual lens elements [see Fig. 1(a)] or by using tubes with thick walls [Fig. 1(b)].

Tube lenses with flat ends are simpler to design and easier to fabricate than elements with stepped ends. This is especially true when they are made from refractory metals, which are difficult to machine. Unfortunately, flat tube ends can allow a direct "line of sight" between the beam and nearby insulators, and are thus not as effective in shielding the electrons from stray fields. The following question then arises: for a given tube gap $g$ [see Fig. 1(b)] between lenses in this flat-end configuration, what must the thickness $t$ of the tube walls be to ensure that charging of the insulators will have a negligible effect on the electron beam trajectories? While we ask this question in the context of electron beam transport by tube lenses, we note that its answer has general applicability as a rule of thumb to any situation in which one wishes to electrostatically isolate two volumes sharing a line of sight.

In putting the above question to a number of the electron

\footnotetext{
a) Author to whom correspondence should be addressed; electronic mail: tander16@bigred.unl.edu
}

optics cognoscenti over the years, the answer appears to be that $t / g$ should be greater than $6 .^{2}$ This rule seems to be followed in the electron optics designs we have seen in other laboratories, although we have not been able to find such a rule codified in the literature. Unfortunately, even for the simplest cylindrical geometry involving two lens elements and an insulator with a uniform charge on its surface, an analytical calculation of the electric fields between the elements appears to be difficult and is not contained in any of the standard texts we consulted covering boundary value problems in electrostatics. Thus we used the powerful threedimensional (3D) program SIMION 3D Version 6 to analyze the problem. ${ }^{3}$

\section{SIMULATED PARAMETERS}

Since we are interested in finding the smallest acceptable aspect ratio $t / g$, we reasoned that we should examine the worst physically reasonable scenario we could imagine. We investigated several configurations, including three common types of cylindrical lens systems. ${ }^{4,5}$ These were an einzel configuration with three elements, a double-element single lens configuration, and a split drift tube. The general shapes of all the insulators in these models were azimuthal segments of a cylindrically symmetric ring that fit between the gaps of the electrodes [Fig. 1(b)]. We found that the einzel configuration with two similarly charged $180^{\circ}$ aligned insulators produced the largest beam deviations. We emphasize that while uniform charging of the same $180^{\circ}$ azimuthal segments of consecutive insulators is physically improbable, it maximizes the effects of charging on beam deviation, and thus represents a "worst possible case" envelope in which actual charging fluctuations would occur. To study single "hot spots" in more realistic situations, we considered $5^{\circ}$ segments.

The einzel lens geometry we studied is shown in Fig. 2. We consider the standard situation in which $g=0.1 D$. The voltages of the tubes are designated as $V_{1}, V_{2}$, and $V_{3}$, with $V_{1}$ corresponding to the "upstream" element. In the simulation, we chose, $V_{1}=V_{3}=+100 \mathrm{~V}$ and $V=+400 \mathrm{~V}$, taking $V_{2} / V_{1}>1$ since this is the most common experimental situation. ${ }^{1}$ (In the double lens system, we used $V_{1}=+100 \mathrm{~V}$ 


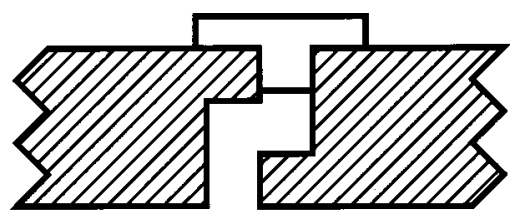

(a)

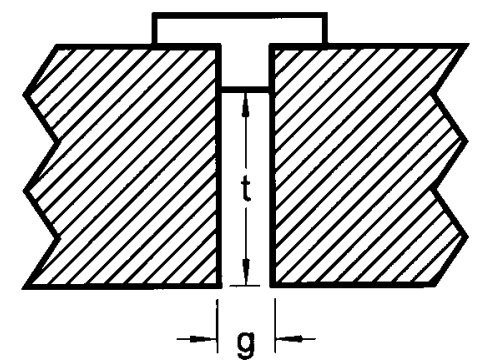

(b)

FIG. 1. (a) Metallic tube lenses (shaded) with "stepped" ends, spaced by insulator. (b) Flat lens ends, showing gap dimensions (see the text).

and $V_{2}=+400 \mathrm{~V}$.) The electron kinetic energy was taken to be $100 \mathrm{eV}$ at the launch point within the first element, corresponding to an (imaginary) source potential of $0 \mathrm{~V}$. Insulators located between the +100 and $+400 \mathrm{~V}$ elements were thus "charged" to $0 \mathrm{~V}$, at which point no electrons could reach the insulator. The $0 \mathrm{~V}$ insulator potential corresponds to the most negative possible value, given a mechanism whereby electrons originating at the source charge the insulators.

In the simulation, the cylindrical electron beam was approximated with 411 separate electron trajectories with an initial kinetic energy of $100 \mathrm{eV}$ and an initial filling factor of $20 \%$. Possible variations in electron source quality were taken into consideration by adjusting the electron beam brightness $B$ at its launch point: ${ }^{6}$

$$
B=\frac{I}{A * \Omega},
$$

where $I$ is the beam current, $A$ is the cross-sectional area of the beam, and $\Omega$ is its opening cone half angle. We considered two values of $B$ in the first lens element: infinity, corresponding to perfectly collimated initial trajectories, and $1.3 \times 10^{-2} \mathrm{~A} \mathrm{~cm}^{-2} \mathrm{sr}^{-1}$, which is typical for a beam ex-

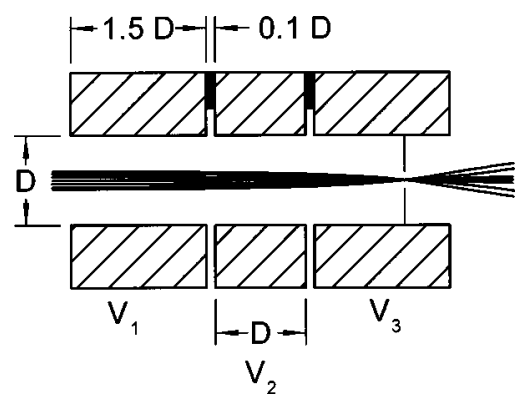

FIG. 2. Cross section of the SIMION einzel configuration geometry. Lens voltages: $V_{1}=V_{3}=+100 \mathrm{~V}$ and $V_{2}=+400 \mathrm{~V}$. The simulation indicated has the insulator "charged" to $0 \mathrm{~V}$. The initial filling factor of the beam is $20 \%$ and the initial kinetic energy is $100 \mathrm{eV}$. The line at the beam waist indicates the virtual aperture (see the text). The radius used for the virtual aperture was $0.00064 \mathrm{D}$.

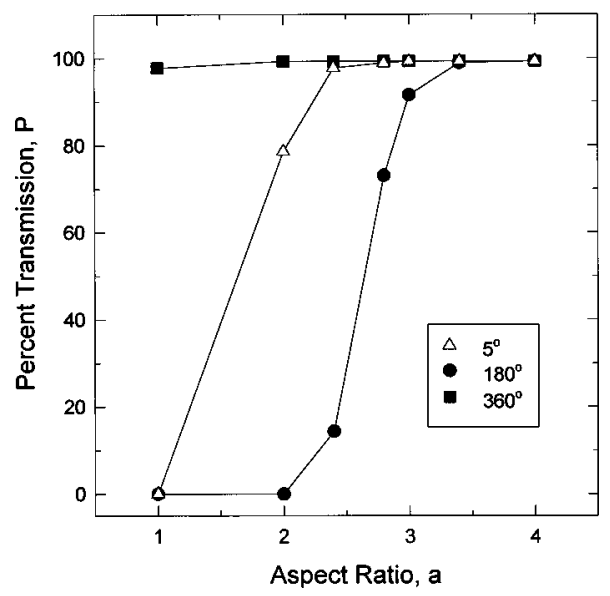

FIG. 3. Percentage beam transmission $P$ through the virtual aperture vs shielding aspect ratio $t / g=a$. The three data sets indicate different azimuthal angular ranges of the insulator segments.

tracted from a hairpin filament. Assuming a typical beam current of $100 \mu \mathrm{A}$ and a lens inner diameter of $4 \mathrm{~cm}$, Eq. (1) yields $\Omega=9.6 \times 10^{-4}$ sr for the hairpin filament.

\section{RESULTS}

After investigating numerous combinations of parameters, we found that the major effect of the insulators was beam deflection rather than changes in the focal properties of the system. As expected, the biggest deflections occurred in the $180^{\circ}$ insulator case. Although $180^{\circ}$ insulator segments charged uniformly combined with beams of infinite brightness are unlikely to occur in real experiments, they follow our criterion of finding the worse possible case. To determine the smallest acceptable aspect ratio, $a=t / g$, we used a somewhat arbitrary but reasonable and quantifiable measure of beam deflection: the percentage of transmission $P$ through a virtual aperture placed at the disk of least confusion ${ }^{1}$ (beam waist) with no insulators in the system. With the infinitely bright electron beam the disk of least confusion, and hence the virtual aperture, was very small. (The nonzero disk of least confusion corresponds to the fact that the electron lens system which was modeled is not aberration free. ${ }^{7}$ ) Any divergence from the central axis thus caused a large drop in $P$, making it a very sensitive indicator of charging effects. The dependence of $P$ on $a$ is illustrated in Fig. 3 for three insulator configurations. Other lens types, voltage ratios, and values of beam brightness produced either similar results or less drastic results than those shown in Fig. 3. Above an aspect ratio of 3 there is less than $10 \%$ beam loss, even in the extreme worst-case scenario.

We emphasize that the data of Fig. 3 represent a worstcase scenario in two ways. First the beam brightness is larger than would be encountered with a real source, so the aperture used to determine $P$ is unrealistically small. Second, the charged insulator segments are aligned and take up an azimuthal arc of $180^{\circ}$ leading to maximum deflection. Using this worst-case scenario as a guide, an aspect ratio of 3 would be appropriate in most situations, and $a=4$ would be a very conservative design parameter. In fact, many lens systems are assembled using thin rods or rings of insulator, 
where the $5^{\circ}$ or $360^{\circ}$ case is more applicable. In these cases, our simulations showed that $10 \%$ of the beam was lost at an aspect ratio of 2.5; therefore it may be possible to use an aspect ratio of 2.5 and still have adequate beam control.

\section{ACKNOWLEDGMENT}

This work was supported by NSF Grant No. PHY9732258.
${ }^{1}$ J. H. Moore, C. C. Davis, and M. A. Coplan, Building Scientific Apparatus, 2nd ed. (Perseus, Reading, PA, 1991).

${ }^{2}$ K. Jost (deceased) and M. H. Kelley (private communication).

${ }^{3}$ D. A. Dahl, Scientific Instrument Services, Inc., Boise Idaho, $<$ http:// www.sisweb.com/simion.htm>.

${ }^{4}$ D. W. O. Heddle, Electrostatic Lens Systems (Adam Hilger, Bristol, 1991).

${ }^{5}$ E. Harting and F. H. Read, Electrostatic Lenses (Elsevier, Amsterdam, 1976).

${ }^{6}$ D. T. Pierce, "Spin-Polarized Electron Sources" in Atomic, Molecular, and Optical Physics: Charged Particles, edited by F. B. Dunning and R. G. Hulet (Academic, San Diego, 1995).

${ }^{7}$ M. Szilagyi, Electron and Ion Optics (Plenum, New York, 1988). 